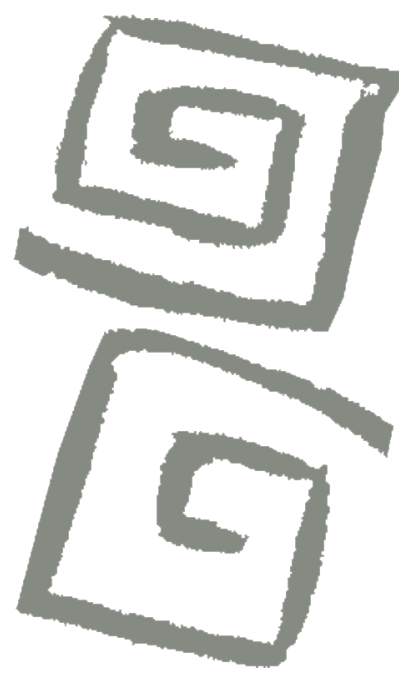

\title{
¿Qué hace un centro de atención psicosocial infanto-juvenil 24 horas en la ciudad de Curitiba, Brasil?
}

\author{
What does a 24-hour child and adolescent psychosocial \\ care center do in the city of Curitiba, Brazil?
}

Deivisson Vianna Dantas dos Santos ${ }^{1}$, Daniele Basegio², Rafael Gomes Ditterich $^{3}$, Isabela Naves Conciani ${ }^{4}$, Sabrina Stefanello ${ }^{5}$

${ }^{1}$ Autor de correspondencia. Doctor en Salud Colectiva. Departamento de Saúde Coletiva, Universidade Federal do Paraná, Curitiba, Paraná, Brasil. $\square$ (iD)

${ }^{2}$ Magíster en Salud Colectiva. Terapista ocupacional, Fundação Estatal de Atenção à Saúde, Curitiba, Paraná, Brasil. $\triangle$ iD

${ }^{3}$ Doctor en Odontología. Departamento de Saúde Coletiva; Universidade Federal do Paraná, Curitiba, Paraná, Brasil. $\triangle$ iD

${ }^{4}$ Médica. Residente de Medicina de Familia y Comunidad, Fundação Estatal de Atenção à Saúde, Curitiba, Paraná, Brasil. $\bowtie$ iD

${ }^{5}$ Doctora en Ciencias Médicas, Departamento de Medicina Forense y Psiquiatría, Universidade Federal do Paraná, Curitiba, Paraná, Brasil. $\triangle$ iD
RESUMEN La política nacional de salud mental de la niñez y la adolescencia aún está mal estructurada en Brasil. En términos generales, se trata de una copia de su equivalente para adultos, lo que dificulta su vinculación con la red. El objetivo de esta investigación fue analizar y caracterizar las acciones desarrolladas en un Centro de Atención Psicosocial Infanto-Juvenil con camas, que atiende las 24 horas del día en la ciudad de Curitiba, Brasil. Se trata de un estudio descriptivo, de análisis de historias clínicas y datos de productividad del año 2016, que fueron provistos por el Departamento de Informática del Sistema Único de Salud (DATASUS). Los resultados muestran que el servicio realizó 29.449 procedimientos, atendió a 112 niñas, niños y adolescentes en la recepción de 24 horas. Las historias clínicas indican el amplio uso del servicio por parte de la red. El 52\% de la demanda provino de la atención primaria, pero la otra mitad de una amplia variedad de servicios. Esa variedad de acciones sugiere una clínica más flexible, que reemplaza el hospital psiquiátrico clásico por los principios de la clínica ampliada. Además, la existencia de camas facilitó la administración de la crisis de forma más vinculada al territorio.

PALABRAS CLAVES Salud Mental; Salud del Niño; Centros Comunitarios de Salud Mental; Brasil.

ABSTRACT The National Policy on Mental Health for Children and Adolescents is still poorly structured in Brazil. In general, it replicates the policy aimed at adults, which creates problems for its articulation with networks. The objective of this study was to analyze and describe the actions carried out at a 24-hour Child and Adolescent Psychosocial Care Center (CAPSi) with beds in the city of Curitiba, Brazil. This is a descriptive study based on the analysis of medical record data and productivity data between 2016-2017, provided by the Department of Informatics at the Unified Health System (DATASUS). Results show that the center performed 29,449 procedures and attended to 112 children and adolescents through its 24-hour service. Medical records indicate extensive use of the service by the network. Primary care accounted for $52 \%$ of demand, while the other half could be attributed to a wide range of services. The variety of actions carried out suggests a high degree of flexibility at the clinic, replacing the classic model of the psychiatric hospital with the principles of the expanded clinic. Furthermore, the existence of beds made it easier to manage crises in a manner more closely linked to the community.

KEY WORDS Mental Health; Child Health; Community Mental Health Centers; Brazil. 


\section{INTRODUCCIÓN}

Este artículo parte de la necesidad de debatir las políticas públicas y la implementación de la Política Nacional de Salud Mental de la Niñez y la Adolescencia, dada la importancia de contar con estrategias que incluyan no solo los centros de atención psicosocial (CAPS), sino la comunicación y la articulación de los diversos equipos de la red de salud involucrados en el cuidado de esta población. La construcción de una política intersectorial permite compartir responsabilidades y saberes, y evitar que los temas complejos recaigan exclusivamente en el campo de la salud. Permite, así, una mirada más amplia sobre problemas como las violencias, las crisis socioeconómicas, desestructuración familiar y sus relaciones con padecimientos mentales ${ }^{(1)}$.

Como lugares de referencia y de cuidado, los CAPS tienen un papel estratégico en la articulación de la Red de Atención Psicosocial (RAPS), tanto en lo que se refiere a la atención directa, que apunta a la promoción de la vida comunitaria y de la autonomía de las personas usuarias, como al cuidado y la atención de situaciones de crisis. Se espera que los CAPS actúen en conjunto con los equipos de salud familiar y los agentes comunitarios de salud, articulando y activando los recursos existentes en otras redes, así como en los territorios.

Para que una persona sea atendida en el CAPS, puede recurrir directamente al servicio, ser derivada por los servicios de atención primaria de la salud (APS) o por cualquier otro servicio de salud. La persona puede concurrir sola o acompañada, preferencialmente, debe dirigirse al CAPS que atiende en la región en la que reside ${ }^{(2)}$.

El Decreto 7508, que reglamenta la Ley 8080, establece que los servicios de APS, las guardias y los servicios de emergencia de atención psicosocial y demás servicios especiales de acceso público son puertas de entrada al sistema de salud ${ }^{(3)}$. Por lo tanto, la APS es el punto de inicio de la atención y la puerta de entrada preferencial ${ }^{(4)}$. Sin embargo, en las recientes resoluciones existen algunas divergencias. En la Resolución 336/02, se asigna a los CAPS una función ordenadora de la red ${ }^{(5)}$; mientras que en la Resolución 3088/11, que instituye la RAPS, los servicios de APS pasan a compartir esa función ${ }^{(6)}$. Estudios sobre la construcción de los servicios de salud mental infanto-juvenil señalan la importancia de la APS en el cuidado de esta población, dado que amplían el acceso tanto en países desarrollados como en desarrollo, por la localización territorial y su carácter menos estigmatizante, en comparación con los servicios especializados ${ }^{(7)}$. A los equipos de APS es donde, en general, llegan los problemas, incluso aquellos relacionados con la niñez y la adolescencia ${ }^{(1)}$.

De esta forma, la atención orientada a la salud mental de niñas, niños y adolescentes y su reconocimiento como un tema de salud pública que forma parte de las acciones del Sistema Único de Salud (SUS) es reciente y ha sido considerada como uno de los principales desafíos de la Reforma Psiquiátrica Brasileña $^{(8)}$. La historia de las políticas de defensa de los derechos de niñas, niños y adolescentes está marcada por el control del Estado sobre esos individuos y la construcción de un modelo de asistencia centrado en la institucionalización, con el objetivo de garantizar la protección social ${ }^{(9)}$. La Reforma Psiquiátrica y las políticas de salud mental infanto-juvenil se insertaron tardíamente en la agenda de la salud pública brasileña, recién a partir de 2001. "En Brasil, dado el enfoque de la salud mental infanto-juvenil, niñas, niños y adolescentes con sufrimiento psíquico, bajo la insignia de la deficiencia, estaban sometidas a la institucionalización en albergues y escuelas de carácter esencialmente adaptativo o correctivo"(10). En 1990, la promulgación del Estatuto de la Niñez y la Adolescencia y la implementación del SUS dieron inicio al proceso de reconocimiento de la niñez y de la adolescencia como sujetos de derechos y responsabilidades, resultando en la elaboración de políticas públicas específicas.

En Brasil, las características de la asistencia infanto-juvenil estuvieron marcadas por estrategias similares a las utilizadas en el 
cuidado de adultos. En la población de niñas, niños y adolescentes, los tipos de trastorno, los principales factores de riesgo y de protección, las estrategias de intervención y de organización del sistema de servicios tienen especificidades que no pueden ser contempladas como una simple extensión de las estrategias de cuidado de la población adulta ${ }^{(1)}$. Entre 2001 y 2018, se promulgaron diversas resoluciones con relación a los CAPS, referentes a los aspectos financieros y presupuestarios y a los procesos de selección para la construcción de CAPS. Solo en cuatro normativas (Resolución 336 del 19 de febrero de 2002, Resolución 3088 del 23 de diciembre de 2011, Resolución 130 del 26 de enero de 2012, Resolución 854 del 22 de agosto de 2012) se explicitaban procedimientos aplicables a la organización del trabajo cotidiano de los CAPS y sobre el tema específico discutido en este artículo, que es el de la salud mental de niñas, niños y adolescentes ${ }^{(5,6,11,12)}$. En las resoluciones, los textos referentes a la salud mental de la población infanto-juvenil parecerían calcados de textos similares destinados a la población adulta, marcando así una carencia en el diseño de políticas específicas para esta población.

Desde la formación del SUS, la estructuración del cuidado en salud mental debería orientarse a la construcción de redes ampliadas e intersectoriales de atención, de base comunitaria, con énfasis en la articulación entre diferentes servicios de atención primaria y especializada. En este sentido, la atención de situaciones de crisis en salud mental cobra importancia en la medida en que la expansión de la RAPS y la disminución de camas psiquiátricas redireccionaron las prácticas de cuidado(13). En la bibliografía brasileña, el término y sus prácticas proponen nuevas acciones, estrategias y cambios en la organización institucional que remiten a la configuración del paradigma de la clínica ampliada $^{(14)}$ en contraposición a un conjunto de discursos y prácticas propios de los hospitales psiquiátricos.

El paradigma de la clínica ampliada fue incorporado a la rehabilitación psicosocial brasileña y al trabajo cotidiano de los servicios de salud mental con conceptos relacionados al vínculo, a la planificación del cuidado en equipo, al conocimiento del contexto del sujeto y respecto a la temporalidad de la crisis; elementos que en el modelo psiquiátrico tradicional se consideraban "extraclínicos"(15). Una de las hipótesis es que la multidisciplinariedad e interdisciplinaridad existentes en los CAPS son responsables tanto de esa ampliación de la clínica como de la articulación de prácticas profesionales diferentes, que generan acciones nuevas y posibilitan el manejo de la crisis en el territorio en el que se inscribe el usuario(16).

Lo que distingue al CAPS analizado en este trabajo es que es uno de los pocos que posee camas 24 hs para el cuidado de niñas, niños y adolescentes en situaciones de crisis, lo cual es un facilitador descripto para la ampliación del carácter substitutivo de los manicomios $^{(13,17)}$. En 2015, el servicio pasó del formato de CAPS II (sin camas) a CAPS III (con camas 24 hs) y hasta hoy es el único equipo de salud mental que cuenta con esta capacidad. Considerando lo que fue expuesto, el objetivo de este estudio fue analizar y caracterizar qué acciones lleva a cabo el Centro de Atención Psicosocial Infanto-Juvenil Boa Vista (CAPSi Boa Vista), e identificar la matriz de cuidados y acciones ofrecidas al público usuario.

\section{MÉTODO}

\section{Caracterización del campo}

El CAPSi Boa Vista contaba en 2016 y 2017 (años de recolección de datos) con cuatro camas para recepción nocturna, caracterizándose como un CAPS infanto-juvenil con funcionamiento 24 hs. El hecho de contar con camas que atendían situaciones agudas y de crisis en el campo de la salud mental, teóricamente permitía aplazar la internación en hospitales psiquiátricos y disminuir la demanda de estos servicios ${ }^{(13)}$. Este CAPSi contaba con 20 trabajadores con nivel educativo superior (título universitario: profesionales en medicina, psicología, enfermería), y 14 
con nivel educativo medio (título técnico en enfermería), pero no recibía apoyo logístico para el transporte de los profesionales en las acciones terapéuticas realizadas en el territorio, como visitas domiciliarias o colegiados entre las redes de atención.

\section{Procedimientos metodológicos}

Se diseñó un estudio descriptivo de análisis de datos secundarios relevados de dos fuentes: 1) las historias clínicas de niñas, niños y adolescentes acogidos en el CAPSi Boa Vista y 2) datos de productividad del sistema de Registro de las Acciones Ambulatorias de Salud (RAAS) y de los Boletines de Producción Ambulatoria Consolidado (BPA-C) e Individualizado (BPA-I), a los que se accedió a través del Departamento de Informática del Sistema Único de Salud (DATASUS). Este tipo de datos secundarios son indicadores de producción tradicionalmente requeridos por el municipio y el padrón nacional.

Se incluyeron las historias clínicas de niñas, niños y adolescentes, clasificados por el equipo del CAPSi Boa Vista, entre febrero y julio de 2016, como "recepción", independientemente de si ya habían sido atendidos previamente en el servicio. Esta clasificación es la que utiliza el servicio para identificar a quienes están siendo atendidos y los que no. Se relevó la totalidad de las historias clínicas generadas a partir de la recepción en el CAPSi y se analizaron todos los registros realizados por el equipo en cada una de las historias clínicas hasta julio de 2017, por lo que se analizó un período de un año de seguimiento (entre julio de 2016 y julio de 2017). Con base en los datos obtenidos, se elaboró un relevamiento de información relacionado con el flujo de la puerta de entrada y origen de las derivaciones del CAPSi Boa Vista. Se enfatizaron los aspectos que emergieron del análisis de las historias clínicas y que eran de interés de esta investigación: origen de la derivación, registros referentes a la relación con la red de salud, estatus del seguimiento en julio de 2017, tanto de los casos recepcionados como del tipo de demanda recibida.
En cuanto a los datos de la RAAS, se analizaron los procedimientos realizados mensualmente por el equipo del CAPSi Boa Vista. Los procedimientos son acciones de salud (individuales o colectivas) o de articulación del cuidado, realizadas por miembros del equipo del CAPSi, y que constan en la Resolución 854 del 22 de agosto de 2012, que amplió la tipificación de procedimientos que podrían ser registrados por los CAPS. Se analizaron, por lo tanto, 16 procedimientos con registro diferente a 0 (cero) que podrían ser realizados en un CAPSi $24 \mathrm{~h}$ - Además de procedimientos como atención individual, grupal o familiar, se analizaron también los procedimientos más recientes incluidos en la Resolución 854, vinculados a las acciones orientadas a una clínica ampliada $^{(14,18)}$ : acciones de articulación del cuidado y apoyo de la atención primaria (matricial), atención de situaciones de crisis, recepción nocturna, articulación con la red intra e intersectorial, atención domiciliaria, prácticas corporales y realización de talleres, entre otras. De esta forma dividimos los procedimientos relevados en tres categorías: a) Acciones que ya existían antes de la Resolución 854 , b) acciones introducidas por la Resolución 854, y acciones que generalmente realiza el propio CAPSi y c) acciones incorporadas por la Resolución 854 y relacionadas con las acciones territoriales y de articulación en red (Tabla 1).

Posteriormente, todos los datos se categorizaron en planillas Excel ${ }^{\circledR}$, y se realizó el análisis descriptivo con números absolutos y porcentajes. En cuanto al análisis de los promedios de permanencias y tasas de ocupación de las camas, por más que sean parámetros tradicionalmente hospitalarios, optamos por utilizar estos parámetros ${ }^{(19)}$ a fin de garantizar la comparabilidad en el análisis de utilización de camas vigentes actualmente.

La investigación fue aprobada por el Comité de Ética en Investigación del Sector de Ciencias de la Salud de la Universidade Federal do Paraná, bajo el número CAEE 62277516.9.0000.0102. 
Tabla 1. Enumeración de los procedimientos realizados en el Centro de Atención Psicosocial Infanto-Juvenil Boa Vista, con relación a la Resolución 854, Curitiba, Brasil, 2016- 2017.

\begin{tabular}{|c|c|}
\hline $\begin{array}{l}\text { Procedimientos con relación a } \\
\text { la Resolución } 854\end{array}$ & Tipos de procedimientos \\
\hline \multirow{4}{*}{$\begin{array}{l}\text { Procedimientos ya existentes } \\
\text { antes de la Resolución } 854\end{array}$} & Atención individual de paciente \\
\hline & Atención en grupos de pacientes \\
\hline & Atención familiar \\
\hline & Atención en psicoterapia \\
\hline \multirow{6}{*}{$\begin{array}{l}\text { Procedimientos menos } \\
\text { tradicionales, pero realizados } \\
\text { en su mayoría en el propio } \\
\text { servicio y oficializados luego de } \\
\text { la Resolución } 854\end{array}$} & Acciones de rehabilitación psicosocial, prácticas de expresión y comunicación \\
\hline & Prácticas corporales y talleres \\
\hline & Atención de situaciones de crisis \\
\hline & Recepción diurna del paciente \\
\hline & Recepción nocturna del paciente \\
\hline & Recepción inicial \\
\hline \multirow{6}{*}{$\begin{array}{l}\text { Procedimientos orientados } \\
\text { a acciones de articulación } \\
\text { y atención en el territorio, } \\
\text { oficializados luego de la } \\
\text { Resolución } 854\end{array}$} & Promoción de contractualidad en el territorio \\
\hline & Acciones de articulación con redes intra e intersectoriales \\
\hline & Atención domiciliaria para pacientes y/o familiares \\
\hline & Organización matricial de la atención básica, de la urgencia y de los servicios hospitalarios \\
\hline & Acciones de reducción de daños \\
\hline & Seguimiento del servicio residencial terapéutico \\
\hline
\end{tabular}

Fuente: Elaboración propia a partir de datos del Departamento de Informática del Sistema Único de Salud (DATASUS).

\section{RESULTADOS}

\section{Análisis de las historias clínicas}

De las 146 historias clínicas analizadas, 32 $(21,9 \%)$ eran usuarios que ya habían sido atendidos en el CAPSi en otro momento y estaban retornando, a los que denominamos "re-recepcionados". De los usuarios evaluados por primera vez, 105 (71,9\%) fueron ingresados al servicio para tratamiento.

Si se consideran las 105 historias clínicas de los usuarios que ingresaron al CAPSi, entre febrero y julio de 2016 , solo $14(13,3 \%)$ se encontraban en seguimiento en julio de 2017 , a $35(33,3 \%)$ se les dio el "alta por abandono de tratamiento" sobre la base de los registros del equipo a lo largo del período; en $14(13,3 \%)$ figuraba "transferencia de cuidados", o sea, fueron derivados para ser tratados en otros lugares (servicios ambulatorios de la red, unidades de salud de referencia y hospitales psiquiátricos). Se percibió que el término "abandono", registrado en las historias clínicas, no sigue un patrón establecido, y puede referir a los casos en que, luego de un tiempo de no asistir al CAPSi e incluso luego de búsquedas activas a través de contactos telefónicos por parte de los equipos del CAPSi o de atención primaria, los usuarios no fueron encontrados. El término "transferencia de cuidados", a su vez, se utilizaba como sinónimo de derivación de los casos a otros servicios.

Desde el momento de la recepción inicial hasta el año de finalización de nuestro análisis, se verificó el registro de mejoras de los síntomas y, como consecuencia, el alta del servicio en 30 casos $(28,6 \%)$. En 5 $(4,8 \%)$, se registró el alta pedido por la familia, sin especificar mejorías y en 7 historias clínicas $(6,7 \%)$ no se encontró ningún registro que indique cómo estaba el tratamiento o su desenlace (Tabla 2).

En cuanto al origen, la mayoría de las derivaciones provenían de los servicios de atención primaria (76 historias clínicas, 52,1\%). 
Tabla 2. Matriz de seguimiento de usuarios que ingresaron por primera vez al Centro de Atención Psicosocial Infanto-Juvenil Boa Vista, Curitiba, Brasil, 2016-2017.

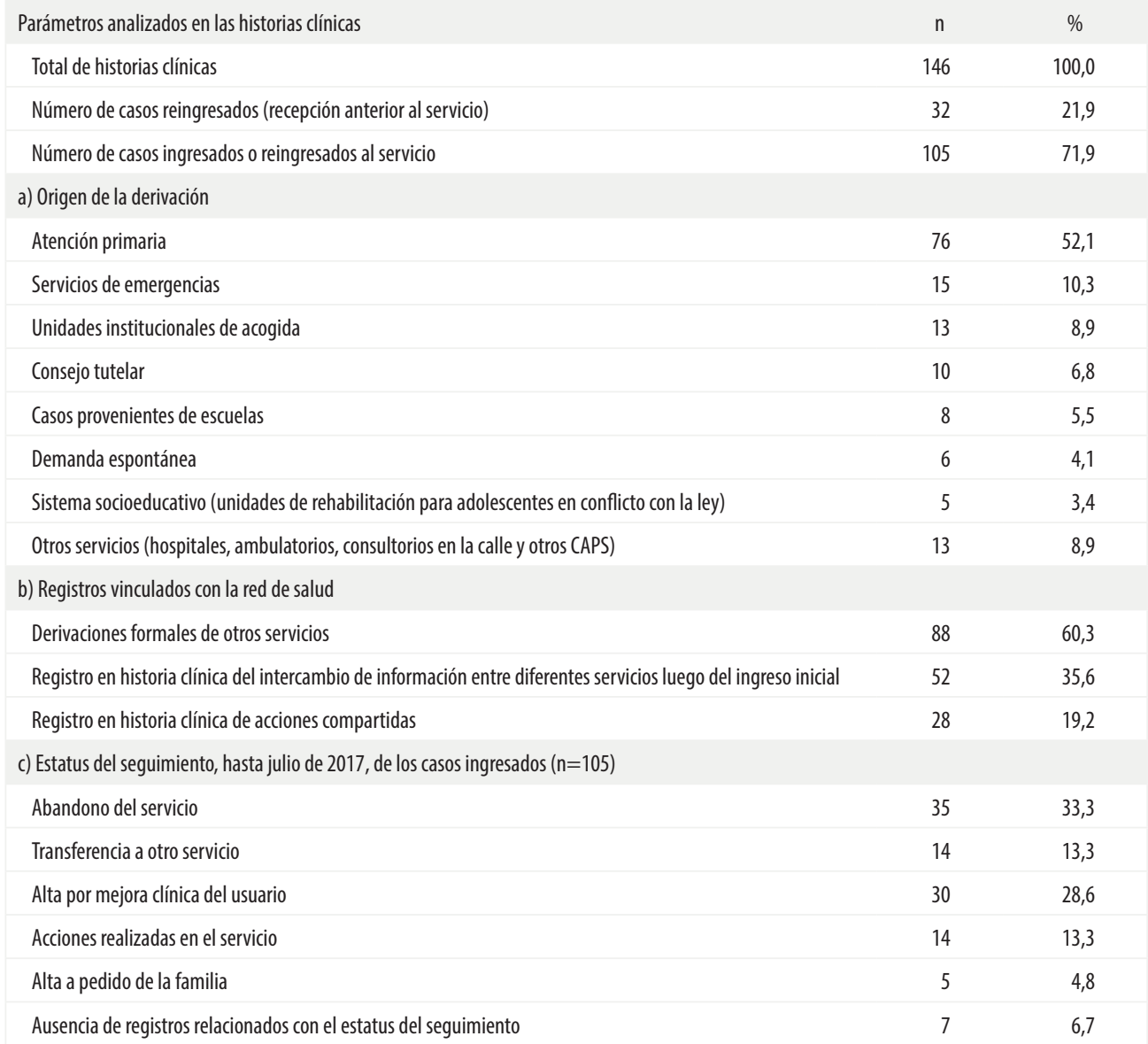

Fuente: Elaboración propia a partir de los datos de las historias clínicas relevadas en el Departamento de Informática del Sistema Único de Salud (DATASUS).

Las demás habían sido solicitadas por servicios de emergencias 24 hs (15 historias clínicas, $10,3 \%$, por unidades institucionales de acogida como, por ejemplo, los albergues (13 historias clínicas, 8,9\%) o mesmo por organismos de fiscalización y defensa del derecho de niñas, niños y adolescentes como los consejos tutelares (10 historias clínicas, $6,8 \%$ ). Otros equipos como escuelas (8 historias clínicas, 5,5\%), unidades del sistema socioeducativos (6 historias clínicas, 4,1\%) y hasta casos por demanda espontánea de la propia familia (5 historias clínicas, 3,4\%). Además, diversos servicios como hospitales, ambulatorios, consultorios en la calle y otros CAPS también funcionaron como fuentes de derivación al CAPSi (13 de las 146 historias clínicas analizadas, 8,9\%) (Tabla 2).
En cuanto al tipo de demanda registrada al momento del ingreso, las más frecuentes fueron las denuncias de agresividad, presentadas por 36 usuarios, y el uso de sustancias psicoactivas por 24 usuarios. Entre las otras demandas registradas se encontraron: intentos de suicidio, conflictos familiares, aislamiento social, agitación, quejas escolares, ideación suicida, automutilación, Ilanto fácil, sospecha o presencia de trastornos del espectro autista, ansiedad, tristeza y depresión.

El $60,3 \%$ de las historias clínicas contenía registros de derivaciones formales, originadas por el servicio que pidió la derivación, con datos sobre el usuario y las razones de la derivación. En las demás historias clínicas, no había ningún tipo de información del servicio que hacía la derivación. Además, 
en 28 historias clínicas $(19,2 \%)$ se observaron anotaciones del equipo sobre acciones como: reuniones colegiadas, discusión de casos en red, visitas domiciliares conjuntas, atención compartida y otras modalidades que indicaban articulación con la red; mientras en $118(80,8 \%)$ no se observaron estas descripciones.

De las 146 historias clínicas analizadas, 11 tenían registros de utilización de las camas 24 hs, por un total de 62 días, lo que da un promedio de permanencia de 5,6 días por cada niña, niño y adolescente. Los motivos de utilización de las camas fueron muy variados, entre ellos: ideación suicida, "brote psicótico", agresividad, uso de sustancias psicoactivas, protección a condiciones que podrían llevar a situaciones de calle, entre otros, sin que existan causas más frecuentes. Es importante destacar que incluso durante el período de ingreso, se registró en la historia clínica que continuaban las acciones de articulación del cuidado como reuniones colegiadas, visitas domiciliares y contactos telefónicos con la red inter e intrasectorial.

\section{Análisis de los datos de productividad}

En 2016, el CAPSi estudiado realizó un total de 29.449 procedimientos, con un promedio de 2.454 por mes (Tabla 3 ). De los procedimientos realizados, el 15,5\% fue de atención individual, el $9,2 \%$ grupal y el $8,3 \%$ a los familiares. Tales procedimientos ya existían antes de la Resolución 854 de 2012, y se refieren a las consultas de diferentes categorías profesionales, estén dirigidas a un usuario de manera individual, a un grupo de usuarios o a sus familiares. El promedio

Tabla 3. Número, promedio y proporción de procedimientos mensuales realizados en el Centro de Atención Psicosocial Infanto-Juvenil Boa Vista, con relación a la Resolución 854, Curitiba, Brasil, 2016.

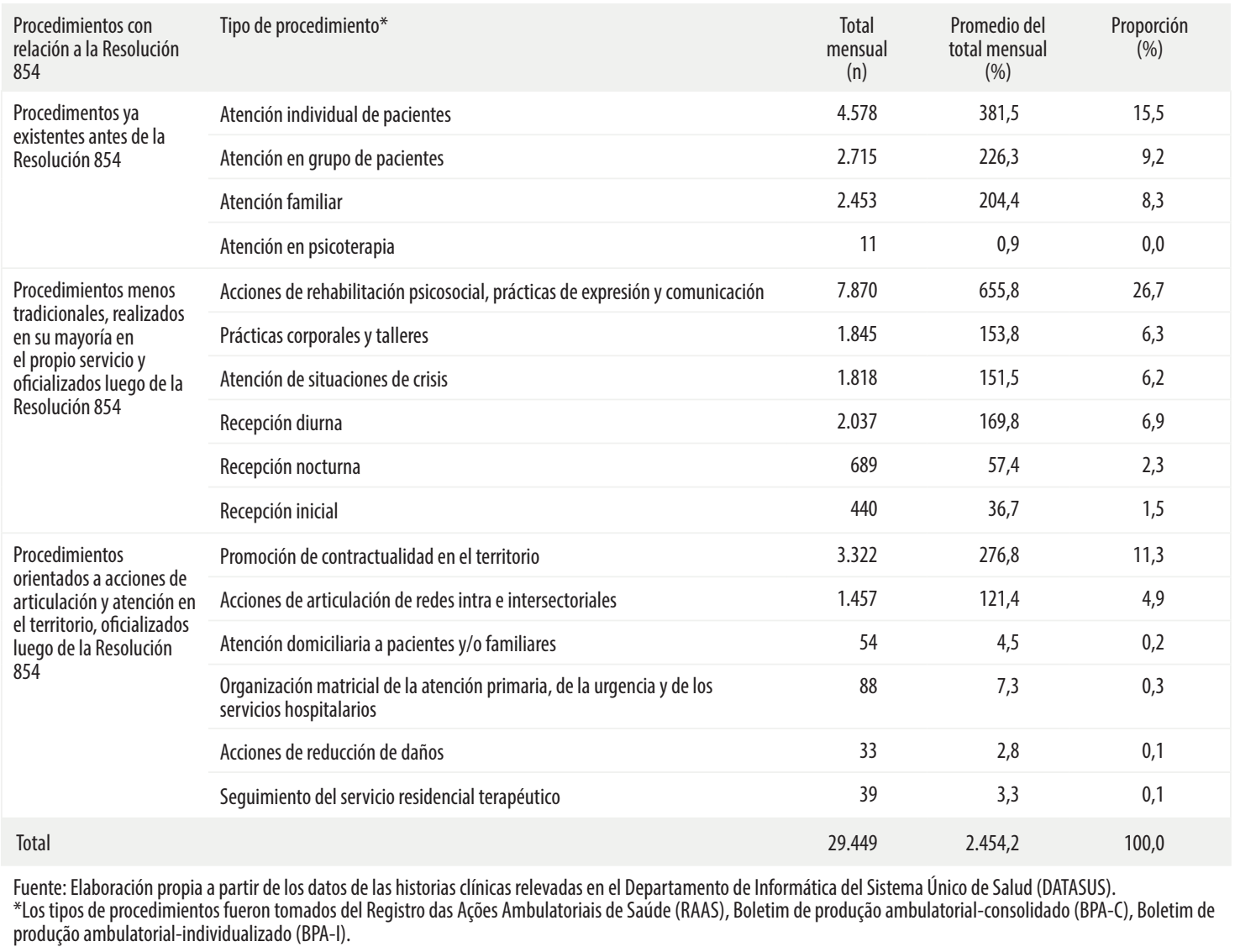


de atención familiar en el CAPSi Boa Vista fue de 204,4 por mes, mientras el total de atención individual, realizadas por los diversos núcleos profesionales, tuvo un promedio mensual de 382,1 consultas. Por otro lado, los porcentajes de los procedimientos menos tradicionales que surgieron luego de la promulgación de la Resolución mencionada fueron el $26,7 \%$ de acciones de rehabilitación psicosocial, el 6,3\% de talleres terapéuticos, y el $6,2 \%$ de prácticas corporales y atención de situaciones de crisis. Así como otras dos acciones que remiten a la atención a la crisis como los "ingresos diurnos y nocturnos" en el CAPSi, $6,9 \%$ y $2,3 \%$, respectivamente.

Al analizar la Tabla 2, otro punto a resaltar es el número de acciones de articulación de red intra e intersectoriales que tuvieron un promedio de 121,4 acciones por mes (4,9\%), así como su registro similar, denominado "promoción de contractualidad en el territorio", que representa el $11,3 \%$ del total. Las acciones más vinculadas a la clínica ampliada y a la articulación del cuidado en el territorio, como las acciones de organización matricial y las visitas domiciliarias, tuvieron un promedio mensual entre 2,8 y 7,3 procedimientos al mes, que representan un $0,5 \%$ de todos los procedimientos realizados por el CAPSi. Otras acciones más territoriales mostraron registros bajos, como "seguimiento de servicio residencial terapéutico" $(0,1 \%)$ y "acciones de reducción de daños" $(0,1 \%)$. Las acciones de organización matricial se definen como la principal forma de articulación de la red con la Resolución 854, a partir de actividades de apoyo presencial sistemático a los equipos de atención primaria, que ofrecen "soporte técnico a la conducción del cuidado en salud mental por medio de discusiones de casos y del proceso de trabajo, atención compartida y acciones intersectoriales en el territorio"(20).

Otro dato importante es sobre la utilización de las cuatro camas de ingreso nocturno del CAPSi 24 hs. Durante 2016, de acuerdo con los datos de productividad, las camas fueron utilizadas por 112 niñas, niños y adolescentes. El total de días utilizados fue de 857 , lo que da un promedio de permanencia de 7,65 días por usuario y una tasa de ocupación del $58,7 \%$ de las camas del servicio durante el año.

\section{DISCUSIÓN}

Entre 2014 y 2015, los CAPS de Curitiba atravesaron cambios en el modelo administrativo. Anteriormente, tercerizaban los servicios a través de diferentes organizaciones no gubernamentales, y luego pasaron a una administración única a cargo de una fundación estatal municipal. Se iniciaron también un conjunto de acciones de reorganización para fortalecer la Política Nacional de Salud Mental, vigente en aquella época, más enfocada en los principios de la Reforma Psiquiátrica Brasileña. Diversos CAPS se ampliaron para garantizar la recepción nocturna, entre ellos, uno destinado al público infanto-juvenil, que es justamente el CAPSi analizado ${ }^{(17)}$.

Uno de los principales hallazgos de este estudio, con énfasis en los datos de productividad, fue la gran variedad de prácticas utilizadas, que sugieren un espectro más amplio y similar a las de la clínica ampliada ${ }^{(14)}$. Los datos encontrados evidenciaron la coexistencia de prácticas convencionales (atención médica y psicoterapéutica) y actividades menos convencionales y territoriales. Tales actividades incluían acciones de atención en grupo, atención de situaciones de crisis, prácticas corporales y expresivas, talleres terapéuticos y acciones de rehabilitación psicosocial. Esta descripción refuerza el carácter del CAPS como equipo de salud que posee un alcance y un formato clínico más amplio de lo que los tradicionales servicios ambulatorios de salud mental. Sin embargo, la mayor parte de las acciones fue realizada dentro de la estructura física del CAPSi, y los datos aún muestran una tímida acción focalizada en la articulación territorial del cuidado. Relatos de experiencias ${ }^{(21,22)}$ muestran la existencia de innumerables posibilidades de combinación y articulación entre los diversos dispositivos y acciones, que generan diferentes configuraciones de redes y resaltan la importancia de esa forma de cuidado. Las diferentes estrategias actualizadas en la construcción 
de la red de atención en salud mental pueden incluir la implementación de servicios substitutivos especializados: implementación de acciones de salud mental en la atención básica; construcción de prácticas como el apoyo matricial, la supervisión de casos y la atención conjunta (realizada entre servicios especializados y de atención primaria); creación de equipos de agentes sanitarios, etc. ${ }^{(23)}$.

En cuanto a las acciones de atención ante situaciones de crisis y el uso de las camas 24 hs, amplían la capacidad de resolución de problemas del servicio. Este hallazgo se alinea con otros estudios que muestran la potencialidad clínica de los CAPS, desde su concepción, con una estructura propia para la articulación de demandas clínicas comple$\mathrm{jas}^{(8,24)}$. Cabe destacar la variedad de motivos encontrados en las historias clínicas no relacionados con los diagnósticos psiquiátricos clásicos. El concepto de crisis en la psiquiatría clásica se circunscribe a la noción de emergencia médica, en la que la crisis implica un desequilibrio y las acciones se orientan a rescatar el equilibrio, a partir de intervenir en el sujeto individual con la utilización de fármacos y contención, excluyéndolo de la convivencia social ${ }^{(25)}$. Desde la lógica de la atención psicosocial, las crisis poseen un enfoque continuo que considera también su territorio, los vínculos socioafectivos de los usuarios y la subjetividad de los propios trabajadores de la salud, lo que posibilita reducir el tiempo de exclusión del entorno ${ }^{(26)}$. Este hecho se visualiza en los bajos promedios de permanencia en los camas del CAPSi, comparativamente con las internaciones psiquiátricas clásicas en Brasil ${ }^{(27)}$.

Por esta razón, desde el paradigma de la Reforma Psiquiátrica Brasileña, la atención de situaciones de crisis debe ser realizada en red, prescindiendo de un servicio especializado único, aún más al verificar que la mayor parte de la demanda por camas proviene de otros servicios y no del propio CAPSi estudiado. Sin embargo, por más que tenga un flujo constante de atención integral para sus camas, el bajo índice de acciones de articulación con otros equipos de salud, limita la concepción de ampliación del cuidado de la crisis en red.
Al observar los datos de las historias clínicas que señalan no adhesión, el 33,3\% de abandono y el $4,8 \%$ de alta a pedido, son bajas y se condicen con las tasas internacionales ${ }^{(28,29,30)}$. Por más que el término "adhesión" remita a un paradigma de atención ambulatoria tradicional, estudios recientes muestran que el abandono del tratamiento en el caso de niñas, niños y adolescentes tiene impactos negativos en la persistencia del problema tratado $^{(31,32)}$. Al mismo tiempo, Anderson et al. ${ }^{(31)}$ y Dossett y Reid ${ }^{(32)}$ señalan que los factores que favorecen una permanencia más duradera y efectiva en el tratamiento se relacionan en forma directa con acuerdos organizacionales, como ingresos más ágiles, con menor tiempo de espera cuando se requiere de atención, procesos administrativos simplificados, relación terapéutica con decisiones compartidas y basadas en las demandas de niñas, niños y adolescentes y apoyo social a las familias. En síntesis, tales acuerdos señalados en la bibliografía internacional coinciden con el abanico de acciones comunes a la lógica de la clínica ampliada existente en los CAPS.

Con relación al origen de las derivaciones, la mitad fue solicitada por los servicios de atención primaria. Souza y Rivera ${ }^{(33)}$ afirman que la atención primaria se coloca como un dispositivo con posibilidad de promoción de otros modos de relacionamiento con la locura, deconstruyendo y construyendo al interior de las casas, en el vecindario, en la comunidad o en el barrio, otras relaciones con las diferencias; permitiendo que la locura ocupe otro espacio de circulación y no aquel "especializado" y segregador. El hecho de que el CAPSi reciba personas, independientemente de la derivación desde la atención primaria, amplía el acceso de los usuarios. Sin embargo, también puede dificultar la conformación de la red, dado que no se crean lazos institucionales entre los servicios. Campos ${ }^{(34)}$, en una investigación evaluativa de una red de salud, describió que la mayor parte de los profesionales de atención primaria, así como los gestores, tienen una visión vaga de las acciones de salud mental en el ámbito de la atención. Adicionalmente, el preconcepto relacionado con la atención 
de problemas de salud mental en la atención primaria puede ser una barrera para la formación de la red, así como la inadecuación de los ambientes formativos y la inexistencia de educación continua que abarque la perspectiva de la inclusión de la atención de la salud mental en las redes locales ${ }^{(35)}$.

Souza ${ }^{(36)}$ afirma que son muchos los desafíos en la implementación de las acciones de salud mental en atención primaria. Entre ellos, destaca la necesidad de formar profesionales para actuar desde una perspectiva menos segregadora y más inclusiva, aprender a dar prioridad a lo más difícil y no restringir la atención tradicional por medio de derivaciones de los casos más graves a otros dispositivos de atención en salud mental. Por otro lado, la entrada al servicio más allá de la atención primaria, o sea, tanto a través de las escuelas, los servicios de emergencias, los equipos de la socioeducación, indica el carácter de puerta abierta propio de los CAPSi ${ }^{(2)}$.

Con relación a la clínica en el territorio, a pesar de ser una práctica minoritaria en las historias clínicas, se identificaron acciones de articulación de red intra e intersectoriales, que indican que existió un diálogo y que hubo conversaciones o reuniones con diversos servicios de la red. Sin embargo, cuando observamos los datos de productividad, se observa un número bajo de acciones compartidas y orientadas al territorio. $\mathrm{Al}$ evaluar los procedimientos, tales acciones son poco realizadas en comparación a las demás. Si consideramos que la intersectorialidad de los servicios de salud es una estrategia importante para el cuidado de niñas, niños y adolescentes, además de fundamental para el cuidado de situaciones de crisis en el territorio, a pesar de su difícil efectivización $^{(15)}$, aumenta la necesidad de realizar investigaciones para comprender los motivos de esta dificultad. De acuerdo con Couto y Delgado $^{(15)}$, la especificidad de esa clínica exige contactos frecuentes con las escuelas, la familia extensa y otros servicios de salud, imponen una articulación de acciones que extrapolan las consultas habituales y necesitan de mucho tiempo fuera del consultorio, lo que muchas veces no es comprendido por la gestión local. Esto tal vez ayude a explicar por qué las acciones de organización matricial, por ejemplo, representan menos del $1 \%$ del total de acciones del servicio.

Por un lado, la organización matricial ya está planteada como una estrategia estructurante y articuladora de la red, dado que ofrece un apoyo asistencial y un soporte técnico-pedagógico a los servicios de referencia, por lo que es un valioso medio para ampliar la clínica ${ }^{(18)}$. Por otro lado, otros estudios señalan que su ejecución es difícil y que tropieza no solo con la dificultad de concepción del trabajo en red -que requiere dejar de lado ciertos conceptos clínicos prestablecidos para construir nuevos con los diferentes equipos de los servicios- sino también por las dificultades logísticas: ausencia de transporte, falta de conocimiento y disponibilidad de los equipos e, incluso, falta de estímulo de la gestión para garantizar la operacionalización de ajustes en las agendas ${ }^{(37,38)}$.

Respecto de las derivaciones y la comunicación entre los servicios, fue llamativa la carencia de registros sobre acciones conjuntas en gran parte de las historias clínicas $(80,8 \%)$. No constaba ningún contacto posterior que informe al equipo o al profesional que realizó la derivación, si la niña o el niño asistieron a la consulta agendada. Los datos de la historia clínica, por lo tanto, contrastan con los datos de productividad que informan lo contrario: un gran número de contactos con la red de servicios en el territorio, con un promedio mensual de 121,4 de acciones de articulación de redes.

Vale destacar que los datos pueden presentar variaciones, dado que no todas las acciones que realizan los profesionales responsables se registran en la historia clínica. Además, otra limitación de la investigación es que los datos de productividad pueden tener subregistros o incluso sufrir presión por parte de la gestión para un sobreregistro de acciones $^{(9)}$. En los servicios comunitarios de salud mental, debido a la complejidad de acciones realizadas, el tiempo necesario para lograr registros de calidad es mayor, por lo que las historias clínicas suelen tener información 
incompleta y simplificada en relación con los relatos reales de los usuarios ${ }^{(39)}$.

\section{CONCLUSIÓN}

A partir de esta investigación, se pudo identificar el origen de las derivaciones al CAPSi Boa Vista, como resultado de los ingresos, las articulaciones de la red durante el seguimiento de un grupo en tratamiento, además del perfil de acciones globales realizadas por el servicio. Tales datos funcionan como piezas de un rompecabezas, el cual daría una visión completa de la red en la que se inserta el CAPSi. Fue posible percibir que, si bien el CAPSi opera como un equipo de clínica amplia, diversa y no tradicional, a pesar de relacionarse con su territorio, esa relación se limita a lo institucional sin fomentar las acciones territoriales. Además, a pesar de que su funcionamiento es menos articulado, presenta tasas de abandono similares a las tasas internacionales, lo que muestra que es posible realizar una clínica no tradicional alineada a la efectividad.

Los CAPSi 24 hs son minoritarios en Brasil y, a pesar de su gran potencial para el manejo y mejores desenlaces de situaciones de crisis en salud mental, son casi inexistentes en el ámbito infanto-juvenil. Para la niñez y la adolescencia, que poseen una clínica aún más conectada a su contexto y a sus realidades familiares y sociales, resulta necesario pensar en posibilidades de cuidado que no se distancien del territorio y que no operen bajo la lógica de la noción de riesgo, de la necesidad de intervención inmediata que permean los ambientes de internación psiquiátricos clásicos. El CAPSi 24 hs estudiado mostró capacidad para conciliar acciones de cuidado continuo de su población objetivo, atención de situaciones de crisis, tanto de sus usuarios adscritos, como de otros CAPSi circundantes.

La demanda identificada justificaría una mayor inversión para la conformación de una red poliárquica con las potencialidades que pueden llegar a tener estos servicios. Sin embargo, vimos cómo las limitaciones institucionales pueden impedir ese potencial, principalmente relacionado con el carácter de articulación en red. Este cuadro se refleja lamentablemente sobre la realidad de la salud mental brasileña actual, donde el subfinanciamiento crónico se alineó al desmantelamiento reciente de las políticas existentes ${ }^{(40)}$.

Sumado a eso, el debate de redes de atención en salud, a partir de las macropolíticas, tiene baja potencia porque no incluye la dimensión micropolítica local y cotidiana. Por eso, se destaca la importancia de reflexionar cómo se da el proceso y los flujos de los usuarios in loco, en los servicios para niñas, niños y adolescentes. En el intento de alcanzar redes menos fragmentadas y que realicen un cuidado de las crisis menos apartado del territorio, es relevante entender cómo los servicios operan los flujos y cómo articulan los dispositivos, levando a una mayor responsabilización de sus prácticas y políticas más efectivas y humanas de cuidado.

\section{REFERENCIAS BIBLIOGRÁFICAS}

1. Ribeiro EL, Tanaka OY. Problemas de saúde mental das crianças: abordagem na atenção básica. São Paulo: Annablume; 2005.

2. Brasil, Ministério da Saúde. Saúde mental no SUS: os centros de atenção psicossocial [Internet]. Brasília: Ministério da Saúde; 2004 [citado 10 mar 2020]. Disponible en: https://tinyurl.com/83wv2uvt.

3. Brasil, Presidência da República. Decreto No. 7.508, de 28 de julho de 2011: Regulamenta a Lei No. 8.080, de 19 de setembro de 1990, para dispor sobre a organização do Sistema Único de Saúde - SUS, o planejamento da saúde, a assistência à saúde e a articulação interfederativa, e dá outras providências [Internet]. 28 jul 2011 [citado 10 mar 2020]. Disponible en: https://tinyurl.com/ ptfz6shc.

4. Giovanella L, Stegmüller K. Crise financeira europeia e sistemas de saúde: universalidade ameaçada? Tendências das reformas de saúde na Alemanha, Reino Unido e Espanha. Cadernos de Saúde Pública. 2014;30:22632281. doi: 10.1590/0102-311X00021314. 
5. Brasil, Ministério da Saúde. Portaria No. 336, de 19 de fevereiro de 2002: Estabelecer que os Centros de Atenção Psicossocial poderão constituir-se nas seguintes modalidades de serviços: CAPS I, CAPS II e CAPS III, definidos por ordem crescente de porte/complexidade e abrangência populacional [Internet]. 19 feb 2002 [citado 10 mar 2020]. Disponible en: https://tinyurl. com/4n6sf95w.

6. Brasil, Ministério da Saúde. Portaria No. 3.088, de 23 de dezembro de 2011: Institui a Rede de Atenção Psicossocial para pessoas com sofrimento ou transtorno mental e com necessidades decorrentes do uso de crack, álcool e outras drogas, no âmbito do Sistema Único de Saúde (SUS) [Internet]. 23 dic 2011 [citado 10 mar 2020]. Disponible en: https://tinyurl.com/2bdvnzfu.

7. Paula CS, Lauridsen-Ribeiro E, Wissow L, Bordin IA, Evans-Lacko $\mathrm{S}$. How to improve the mental health care of children and adolescents in Brazil: actions needed in the public sector. Brazilian Journal of Psychiatry. 2012;34(3):334-351. doi: 10.1016/j.rbp.2012.04.001.

8. Reis AO, Delfini PS, Dombi-Barbosa C, Oliveira M Lauridsen-Ribeiro E, Tanaka OY. Crianças e adolescentes em sofrimento psíquico atendidos nos centros de atenção psicossocial infanto-juvenis. En: LauridsenRibeiro E, Tanaka OY, comp. Atenção em saúde mental para crianças e adolescentes no SUS. São Paulo: Hucitec; 2010. p. 186-210.

9. Neves TCCL, Montenegro LAA, Bittencourt SDA. Produção e registro de informações em saúde no Brasil: panorama descritivo através do PMAQ-AB. Saúde em Debate. 2014;38:756-770.

10. Couto MCV. Por uma política pública de saúde mental para crianças e adolescentes. En: Ferreira T, Paez Varela A. A criança e a saúde mental: enlaces entre a clínica e a política. Belo Horizonte: Autêntica; 2004. p. 61-72.

11. Brasil, Ministério da Saúde. Portaria No. 130 de 26 de janeiro de 2012: Redefine o Centro de Atenção Psicossocial, Álcool e outras Drogas 24 horas (CAPS AD III) e os respectivos incentivos financeiros [Internet]. 26 ene 2012 [citado 10 mar 2020]. Disponible en: https:// tinyurl.com/habcy9n4.

12. Brasil, Ministério da Saúde. Portaria No. 854 de 22 de agosto de 2012 [Internet]. 22 ago 2012 [citado 10 mar 2020]. Disponible en: https://tinyurl.com/d47uzbw8.

13. Dias MK, Ferigato SH, Fernandes ADSA. Atenção à crise em saúde mental: centralização e descentralização das práticas. Ciência \& Saúde Coletiva. 2020;25:595602. doi: 10.1590/1413-81232020252.09182018.

14. Campos GWS, Amaral MA. A clínica ampliada e compartilhada, a gestão democrática e redes de atenção como referenciais teórico-operacionais para a reforma do hospital. Ciência \& Saúde Coletiva. 2007;12(4):849859. doi: 10.1590/S1413-81232007000400007.

15. Couto MCV, Delgado PGG. Presença viva da saúde mental no território: construção da rede pública ampliada de atenção para crianças e adolescentes. En:
Lauridsen-Ribeiro E, Lykouropoulos CB, org. O Capsi e o desafio da gestão em rede. São Paulo: Hucitec; 2016. p. 161-192.

16. Anjos Filho NC, Souza AMP. A percepção sobre o trabalho em equipe multiprofissional dos trabalhadores de um Centro de Atenção Psicossocial em Salvador, Bahia, Brasil. Interface-Comunicação, Saúde, Educação. 2017:21:63-76. doi: 10.1590/1807-57622015.0428.

17. Dias MK, Schlotag AC. A reorganização da Rede de Saúde Mental de Curitiba. En: Moysés SJ, Dercy Filho A, org. A gestão participativa na saúde de Curitiba. Curitiba: Instituto Municipal de Administração Pública; 2016. p. 75-79.

18. Campos GWS, Domitti AC. Apoio matricial e equipe de referência: uma metodologia para gestão do trabalho interdisciplinar em saúde. Cadernos de Saúde Pública. 2007;23(2):399-407. doi: 10.1590/S0102$311 \times 2007000200016$.

19. Brasil, Ministério da Saúde. Portaria No. 2395, de 11 de outubro de 2011: Dispõem sobre a organização do componente hospitalar da rede de atenção às urgências no âmbito do Sistema Único de Saúde [Internet]. 2011 [citado 10 mar 2020]. Disponible en: https://tinyurl. com/37bcf7eh.

20. Ministério da Saúde, Conselho Nacional do Ministério Público. Atenção psicossocial a crianças e adolescentes no SUS: tecendo redes para garantir direitos. Brasília: Ministério da Saúde, Conselho Nacional do Ministério Público; 2012.

21. Souza PJC. Resposta à crise: a experiência de Belo Horizonte. Política de Saúde Mental de Belo Horizonte: O Cotidiano de uma Utopia. En: Lancetti A, Campos FB, org. Experiências da Reforma Psiquiátrica. São Paulo: Hucitec; 2010. p. 101-123

22. Oliveira $M$, Mendonça J. CAPSi e a rede ampliada: Intersetorialidade. En: Lauridsen-Ribeiro E, Lykouropoulos $C B$, org. O Capsi e o desafio da gestão em rede. São Paulo: Hucitec; 2016. p. 193-207.

23. Zambenedetti G, Perrone CM. O processo de construção de uma rede de atenção em Saúde Mental: desafios e potencialidades no processo de Reforma Psiquiátrica. Physis: Revista de Saúde Coletiva. 2008;18(2):277-293. doi: 10.1590/S0103-73312008000200005

24. Ronchi JP, Avellar LZ. Saúde mental da criança e do adolescente: a experiência do Capsi da cidade de Vitória-ES. Psicologia: Teoria e Prática. 2010;12(1):71-84.

25. Jardim KF, Dimenstein MD. Risco e crise: pensando os pilares da urgência psiquiátrica. Psicologia em Revista. 2007;13(1):169-190

26. Ferigato SH, Campos RTO, Ballarin MLGS. O atendimento à crise em saúde mental: ampliando conceitos. Revista de Psicologia da UNESP. 2007;6(1):31-44.

27. Lara APM, Volpe FM. Evolução do perfil das internações psiquiátricas pelo Sistema Único de Saúde em Minas Gerais, Brasil, 2001-2013. Ciência \& Saúde Co- 
letiva. 2019;24(2):659-668. doi: 10.1590/1413-812320 18242.14652017.

28. Verdoux H, Lengronne J, Liraud F, Gonzales B, Assens F, Abalan F, et al. Medication adherence in psychosis: predictors and impact on outcome: A 2-year follow-up of first-admitted subjects. Acta Psychiatrica Scandinavica. 2000;102(3):203-210. doi: 10.1034/j.16000447.2000.102003203.x.

29. de Haan AM, Boon AE, de Jong JTVM, Hoeve $M$, Vermeiren RRIM. A meta-analytic review on treatment dropout in child and adolescent outpatient mental health care. Clinical Psychology Review. 2013;33(5):698-711. doi: 10.1016/j.cpr.2013.04.005.

30. Edbrooke-Childs J, Boehnke JR, Zamperoni V, et al. Service- and practitioner-level variation in non-consensual dropout from child mental health services. European Child \& Adolescent Psychiatry. 2020;29:929-934.

31. Anderson JK, Howarth E, Vainre $M$, Jones PB, Humphrey A. A scoping literature review of servicelevel barriers for access and engagement with mental health services for children and young people. Children and Youth Services Review. 2017;77:164-176. doi: 10.1016/j.childyouth.2017.04.017.

32. Dossett KW, Reid GJ. Predicting dropout from children's mental health services: using a need-based definition of dropout. Child Psychiatry \& Human Development. 2019;51(1):13-26. doi: 10.1007/s10578-01900906-4.

33. Souza A, Rivera FJU. A inclusão das ações de saúde mental na Atenção Básica: ampliando possibilidades no campo da saúde mental. Tempus Actas Saúde Coletiva. 2010;4(1):105-114.
34. Campos RTO, Furtado JP, Passos E, Ferrer AL, Miranda L, Gama CAP da. Avaliação da rede de centros de atenção psicossocial: entre a saúde coletiva e a saúde mental. Revista de Saúde Pública. 2009;43:16-22. doi: 10.1590/S0034-89102009000800004.

35. Gazignato ECS, Silva CRC. Saúde mental na atenção básica: o trabalho em rede e o matriciamento em saúde mental na Estratégia de Saúde da Família. Saúde em Debate. 2014;38:296-304.

36. Souza A. Estratégias de inclusão da saúde mental na atenção básica: um movimento das marés. São Paulo: Hucitec; 2015.

37. Silva DS. Apoio matricial em saúde mental: uma análise sob ótica dos profissionais de saúde da atenção primária. Revista Portuguesa de Enfermagem de Saúde Mental. 2011;(6):20-27. doi: 10.19131/rpesm.0084.

38. Ferreira TPS, Sampaio J, Souza ACN, Oliveira DL, Gomes LB. Produção do cuidado em Saúde Mental: desafios para além dos muros institucionais. InterfaceComunicação, Saúde, Educação. 2016;21:373-384. doi: 10.1590/1807-57622016.0139.

39. Tsai J, Bond G. A comparison of electronic records to paper records in mental health centers. International Journal of Quality in Health Care. 2008;20(2):136-143. doi: 10.1093/intqhe/mzm064.

40. Cruz NFO; Gonçalves RW; Delgado PGG. Retrocesso da reforma psiquiátrica: o desmonte da política nacional de saúde mental brasileira de 2016 a 2019. Trabalho, Educação e Saúde. 2020;18(3):1-20. doi: 10.1590/1981-7746-sol00285. 